\title{
Qualidade da dieta de jovens aos 18 anos de idade, pertencentes à coorte de nascimentos de 1993 da cidade de Pelotas (RS), Brasil
}

\author{
Quality of the diet of 18-year-old adolescents \\ belonging to the birth cohort of 1993 in Pelotas \\ in the State of Rio Grande do Sul, Brazil
}

Cristina Bossle de Castilhos ${ }^{1}$
Bruna Celestino Schneider ${ }^{2}$
Ludmila Correa Muniz $^{3}$
Maria Cecília Formoso Assunção ${ }^{3}$
${ }^{1}$ Departamento de Saúde Pública, Prefeitura Municipal de Pelotas. Av. Visconde da Graça 107, Simões Lopes. 96025-370 Pelotas RS Brasil.cristinacastilhos@ hotmail.com

${ }^{2}$ Departamento de Medicina Social, Universidade Federal de Pelotas (UFPel).

3 Faculdade de Nutrição, UFPel.

\begin{abstract}
The scope of this article is to describe the quality of the diet of adolescents according to sociodemographic and behavioral factors. It involved a cross-sectional study with 3,959 eighteenyear-old adolescents belonging to the birth cohort of 1993 in Pelotas in the State of Rio Grande do Sul, Brazil. Dietary intake was assessed using a semi quantitative Food Frequency Questionnaire with a recall period of 12 months. The diet quality was assessed using the Diet Quality Index Revised (DQI-R). This index ranges from 0 to 100 points and the higher the score, the better the diet quality. The overall DQI-R mean score was 62.4 points (DP). The lowest rates were observed for dark green and orange vegetables (3.0), total vegetables (3.4) and dairy products (3.6). Non-Caucasian adolescents (63.1), from families in which the heads of the household had less schooling (63.6) and belonging to the lowest quintile of the asset index (64.7) had higher average scores in the DQI-R. Lower averages were found among adolescents who smoked (58.5) and who consumed alcoholic beverages (56.0). This study showed that diet quality of the adolescents assessed deserve attention, especially with regard to the intake of vegetables, milk and dairy products.
\end{abstract}

Key words Adolescent, Diet quality index, Diet quality, Nutrition, Food intake
Resumo O objetivo deste artigo é descrever a qualidade da dieta de adolescentes segundo fatores sociodemográficos e comportamentais. Estudo transversal com 3.959 adolescentes de dezoito anos de idade, pertencentes à coorte de nascimentos de 1993, de Pelotas, Rio Grande do Sul, Brasil. O consumo alimentar foi avaliado através de um Questionário de Frequência Alimentar semiquantitativo, com periodo recordatório de 12 meses. A qualidade da dieta foi avaliada através do Índice de Qualidade da Dieta Revisado (IQD-R). Este indice varia de 0 a 100 pontos e quanto maior a pontuação, melhor a qualidade da dieta. A média geral do escore do IQD-R foi de 62,4 pontos (DP 12). Adolescentes com cor da pele não branca $(63,1)$, provenientes de familias cujos chefes tinham menor escolaridade $(63,6)$ e pertencentes ao menor quintil do indice de bens $(64,7)$ apresentaram maiores médias no escore do IQD-R. Menores médias foram encontradas entre adolescentes que fumavam $(58,5)$ e que consumiam bebida alcoólica $(56,0)$. Este estudo mostrou que a qualidade da dieta dos adolescentes avaliados merece atenção, especialmente no que se refere à ingestão de vegetais, leites e derivados.

Palavras-chave Adolescente, Índice de qualidade da dieta, Qualidade da dieta, Nutrição, Consumo alimentar 


\section{Introdução}

A adolescência, período de transição entre a infância e a vida adulta, corresponde à faixa etária de 10 a 19 anos e 11 meses e é caracterizada por intensas transformações físicas e biológicas. Durante a adolescência, o indivíduo adquire $25 \%$ da sua estatura final e $50 \%$ do seu peso definitivo ${ }^{1}$. Hábitos e aprendizagens deste período repercutem sobre muitos comportamentos da vida futura, como alimentação, autoimagem, saúde individual, valores, preferências e desenvolvimento psicossocial $^{2}$.

Em nível mundial, os adolescentes representam aproximadamente $20 \%$ da população ${ }^{1}$. No Brasil, segundo dados do Instituto Brasileiro de Geografia e Estatística (IBGE), no ano de 2010, a população total de adolescentes era pouco mais de 34 milhões de indivíduos que representavam $17 \%$ da população total ${ }^{3}$.

Estudos recentes têm identificado, neste grupo etário, hábitos alimentares pouco saudáveis, especialmente entre os jovens pertencentes às classes econômicas mais favorecidas ${ }^{2}$. Estes jovens, que possuem maior acesso aos alimentos e à informação ${ }^{4}$, adotam frequentemente dietas ricas em gorduras, açúcares e sódio, com pequena participação de frutas e hortaliças ${ }^{5}$. Observa-se, ainda entre eles, consumo menos frequente de alimentos como arroz e feijão ${ }^{6}$.

De acordo com Fisberg et al. ${ }^{7}$, a análise das características da dieta com suas diversas combinações de alimentos, mais do que o consumo de itens alimentares específicos ou a ingestão de nutrientes, pode ser útil, já que os alimentos não são consumidos isoladamente e refletem a escolha por determinado estilo de vida. Por isso, torna-se conveniente a utilização de índices que avaliam a ingestão global de alimentos e nutrientes e que incluam aspectos da dieta simultaneamente. Os índices de qualidade da dieta têm sido desenvolvidos para a obtenção de uma medida resumo das principais características da alimentação, facilitando a avaliação da qualidade desta em populações ou grupos de indivíduos ${ }^{8}$.

Previdelli et al. ${ }^{9}$ adaptaram e validaram para a população brasileira o Índice de Qualidade da Dieta Revisado (IQD-R), tendo por base o Health Eating Index (HEI) $2005^{10}$. Este índice avalia uma combinação de diferentes tipos de alimentos, nutrientes e constituintes da dieta em relação às recomendações dietéticas do Guia Alimentar para a População Brasileira ${ }^{11}$.

Sabe-se que os hábitos alimentares adquiridos durante a adolescência poderão perpetuar-se na vida adulta ${ }^{12}$. Assim, torna-se necessário conhecer a qualidade global da dieta consumida pelos jovens e, desta forma, obter informações que possam nortear a realização de intervenções precoces, se necessárias. Portanto, o objetivo do presente estudo é avaliar a qualidade da dieta de adolescentes com 18 anos de idade, pertencentes a uma coorte de nascimentos de Pelotas/RS a partir do cálculo do IQD-R.

\section{Métodos}

Este é um estudo transversal descritivo aninhado à coorte de nascimentos de 1993 da cidade de Pelotas, Rio Grande do Sul, Brasil. Essa coorte de nascimentos faz parte de um estudo de base populacional que recrutou, no ano de 1993, todos os nascidos vivos residentes na zona urbana de Pelotas e acompanhou em diferentes momentos subamostras ou todos os indivíduos que faziam parte do estudo. Detalhes sobre os acompanhamentos anteriores são encontrados em outras publicações $^{13-15}$

Os dados utilizados neste estudo são referentes ao último acompanhamento dos nascidos em 1993, que aconteceu entre os meses de setembro de 2011 e abril de 2012, quando os adolescentes estavam com 18 anos de idade.

Das 5.249 crianças nascidas vivas em 1993, 164 foram detectadas como óbitos e 4106 foram entrevistadas aos 18 anos, representando 81,3\% da coorte original ${ }^{15}$.

Para a construção do IQD-R foram utilizadas informações de consumo alimentar coletadas através de um questionário de frequência alimentar (QFA) semiquantitativo, composto por 88 alimentos, com período recordatório de um ano. Esse questionário foi autoaplicado, em formato eletrônico, nas dependências da clínica do Centro de Pesquisas Epidemiológicas e na presença de uma monitora. Havia uma sala específica para aplicação do QFA com seis computadores.

As demais variáveis utilizadas foram sexo (coletado no estudo perinatal), cor da pele (autorreferida), índice de bens (em quintis), escolaridade do adolescente, escolaridade do chefe da família no momento do acompanhamento (ambas em anos completos de estudo na escola), estado nutricional avaliado através do Índice de Massa Corporal (IMC - peso em Kilogramas dividido pela altura em metros quadrados), hábito atual de fumar (pelo menos uma vez por semana) e consumo prejudicial de álcool baseado no questionário do The Alcohol Use Disorders Iden- 
tification Test AUDIT, com ponto de corte igual ou superior a 8 pontos $^{16}$.

O índice de bens foi desenvolvido a partir da posse de doze bens e da escolaridade do chefe da família, por meio de análise de componentes principais ${ }^{17}$. Os bens utilizados para o desenvolvimento deste indicador no acompanhamento dos 18 anos foram: aparelho de televisão colorida, aparelho de televisão de LCD ou plasma, automóvel, aspirador de pó, DVD, video game, máquina de lavar roupa, forno de microondas, geladeira, freezer, linha telefônica, computador de mesa, notebooks, internet disponível 24 horas, aparelho de ar condicionado ou split, número de peças da casa utilizadas para dormir, número de banheiros, se possui outro imóvel e empregada doméstica mensalista.

O IMC foi calculado a partir do peso e altura. A altura foi obtida com o auxílio de estadiômetro desmontável (alumínio e madeira) precisão 0,1 $\mathrm{cm}$ e o peso através da balança acoplada à cabine pletismográfica BOD POD ${ }^{\circledast}$. O estado nutricional do adolescente foi classificado em baixo peso, eutrófico, sobrepeso e obeso, de acordo com os pontos de corte recomendados pela Organização Mundial da Saúde, sendo utilizados os percentis referentes ao ponto médio da idade e categorizados em duas classes: com e sem excesso de peso.

A qualidade da dieta foi avaliada através do IQD-R ${ }^{9}$ adaptado para a população brasileira. Este índice é calculado a partir de pontuação atribuída entre os componentes que caracterizam a dieta, sendo o valor máximo de 100 pontos. Quanto maior a pontuação, melhor a qualidade da dieta.

Para o cálculo do IQD-R os alimentos e bebidas contidos no QFA foram organizados em dez componentes, sendo sete baseados no consumo diário de grupos de alimentos conforme o Guia Alimentar para a População Brasileira ${ }^{11}$ : cereais totais; leite e derivados; frutas totais; vegetais totais; vegetais verdes-escuros e alaranjados; carnes, ovos e leguminosas; óleos, oleaginosas e gordura de peixe. Dois componentes baseados em nutrientes (sódio e gordura saturada) e um nos itens dietéticos: Gordura sólida saturada e trans, Álcool e Açúcar de adição (Gord-AA).

Para cada item alimentar, os participantes foram questionados quanto à frequência de consumo e quantidade consumida nos últimos 12 meses. Oito opções de respostas em relação à frequência de consumo foram dadas: nunca ou $<1$ vez/mês; $1-3$ vezes/mês; 1 vez/semana; $2-4$ vezes/ semana; 5-6 vezes/semana; 1 vez/dia; $2-4$ vezes/ dia e $\geq 5$ vezes/dia. Foi efetuada a transformação de todas as frequências em diárias, sendo atribuído o valor 1,0 ao consumo de uma vez ao dia, e aplicados valores proporcionais para as demais frequências. Tais valores foram: zero para alimentos consumidos menos de uma vez ao mês; 0,067 para alimentos consumidos uma a três vezes por mês; 0,143 para alimentos consumidos uma vez por semana; 0,429 para alimentos consumidos 2 a 4 vezes na semana; 0,786 para alimentos consumidos 5 a 6 vezes por semana; 1 para alimentos consumidos uma vez ao dia; 3 para alimentos consumidos três vezes ao dia e 5 para alimentos consumidos cinco vezes ou mais por dia. A quantidade diária consumida de cada item foi obtida a partir da multiplicação da frequência diária pela porção média consumida.

Para o cálculo do consumo calórico dos adolescentes foi utilizada uma planilha do programa Excel ${ }^{\circledR}$, onde foram atribuídos valores correspondentes à composição nutricional de cada um dos alimentos contidos no QFA, baseados na Tabela Brasileira de Composição de Alimentos $(\mathrm{TACO})^{18}$ e na tabela do Departamento de Agricultura Norte Americano - United States Department of Agriculture (USDA), 2003.

O IQD-R desenvolvido por Previdelli ${ }^{9}$ foi modificado para utilização neste estudo. $\mathrm{O}$ item "Cereais integrais" não foi contemplado por haver somente a distinção de um tipo de cereal integral (pão integral) no QFA utilizado. Foram então consideradas três porções de cereais em 1000 Kcal equivalendo a 10 pontos como critério para pontuação máxima neste item. Em relação aos componentes "frutas totais" e "frutas integrais", optou-se por agrupá-los, somando os pontos dos dois componentes, pois no QFA só havia uma opção de suco natural (fruta ou polpa). Assim, o consumo de 1,5 porções em $1.000 \mathrm{Kcal}$ de frutas ou sucos naturais ao dia equivale à pontuação máxima de 10 pontos.

No componente "óleos, oleaginosas e gordura de peixe" não foi computado o óleo vegetal utilizado na culinária, pois, o mesmo não estava contemplado no QFA.

A pontuação máxima para cada componente ficou assim distribuída: 5 pontos ("Vegetais Totais; Vegetais Verdes Escuros e Alaranjados e Leguminosas"), 10 pontos ("Cereais Totais"; "Frutas Totais"; "Leite e derivados"; "Carne, Ovos e Leguminosas"; "Óleos"; “Gordura Saturada” e "Sódio") e 20 pontos ("Calorias Provenientes da Gordura Sólida, saturada e trans, Álcool e Açúcar de Adição - Gord_AA”), conforme demonstrado no Quadro 1. 


\begin{tabular}{|c|c|c|c|c|c|}
\hline \multirow[t]{13}{*}{$\begin{array}{l}\vec{J} \\
\tilde{J} \\
0 \\
0 \\
\infty \\
0 \\
\text { 营 } \\
\tilde{J}\end{array}$} & $\begin{array}{l}\text { Quadro 1. Descrição das pontu } \\
\text { respectivas porçôes. }\end{array}$ & coes dos compone & es do Índice de & lidade da Dieta Revisad & D-R) e \\
\hline & \multirow{2}{*}{ Componentes } & \multirow{2}{*}{$\begin{array}{l}\text { Critério para } \\
\text { pontuação } \\
\text { mínima }\end{array}$} & \multirow{2}{*}{$\begin{array}{l}\text { Critério para } \\
\text { pontuação } \\
\text { intermediária }\end{array}$} & \multicolumn{2}{|c|}{ Critério para pontuação máxima } \\
\hline & & & & Porções & Pontos \\
\hline & Vegetais Totais & \multirow{7}{*}{$\begin{array}{l}\text { Ausência de } \\
\text { Consumo }\end{array}$} & \multirow{7}{*}{$\begin{array}{c}\text { Cálculo } \\
\text { Proporcional }\end{array}$} & 1,0 porção/1000Kcal & \multirow{2}{*}{$\begin{array}{c}5 \\
\text { Pontos }\end{array}$} \\
\hline & $\begin{array}{l}\text { Vegetais Verdes Escuros } \\
\text { e Alaranjados e Leguminosas }\end{array}$ & & & 0,5 porção/1000Kcal & \\
\hline & Frutas totais & & & 1,5 porção/1000Kcal & \multirow{7}{*}{$\begin{array}{c}10 \\
\text { Pontos }\end{array}$} \\
\hline & Cereais Totais & & & 3 porções/1000Kcal & \\
\hline & Leite e derivados & & & 1,5 porções/1000Kcal & \\
\hline & Carnes, Ovos e Leguminosas & & & 1,0 porção/1000Kcal & \\
\hline & Óleos & & & 0,5 porção/1000Kcal & \\
\hline & Gordura Saturada & $\begin{array}{c}\geq 15 \% \text { do } \mathrm{VET}^{\mathrm{a}} \\
0 \text { pontos }\end{array}$ & $\begin{array}{l}10 \% \text { do VET } \\
8 \text { pontos }\end{array}$ & $\leq 7 \%$ do VET & \\
\hline & Sódio & $\begin{array}{l}\geq 2,0 \mathrm{~g} / 1000 \mathrm{Kcal} \\
0 \text { pontos }\end{array}$ & $\begin{array}{l}1 \mathrm{~g} / 1000 \mathrm{Kcal} \\
8 \text { pontos }\end{array}$ & $\leq 0,75 \mathrm{~g} / 1000 \mathrm{Kcal}$ & \\
\hline & Gord_AA ${ }^{\mathrm{b}}$ & $\begin{array}{c}\geq 35 \% \text { do VET } \\
0 \text { pontos }\end{array}$ & $\begin{array}{c}\text { Cálculo } \\
\text { proporcional }\end{array}$ & $\leq 10 \%$ do VET & $\begin{array}{c}20 \\
\text { Pontos }\end{array}$ \\
\hline
\end{tabular}

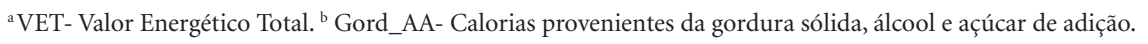

Os dados foram analisados no programa STATA 12.1 (StataCorp, College Station, Texas, EUA). Foram realizadas análises descritivas para caracterizar a amostra estudada e análises bivariadas entre essas características e as médias do IQD-R, através de ANOVA e teste $t$ de student para variáveis dicotômicas, considerando um nível de significância de 5\%.

O protocolo do estudo foi aprovado pelo Comitê de Ética em Pesquisa da Faculdade de Medicina, Universidade Federal de Pelotas, e a assinatura do Termo de Consentimento Livre e Esclarecido (TCLE) foi obtida antes de cada entrevista.

\section{Resultados}

Dos 4.106 jovens entrevistados aos 18 anos, foram objeto deste estudo 3.959, sendo excluídos os individuos cujo consumo alimentar foi considerado pouco plausível: consumo cujo valor calórico diário era maior que $3 \mathrm{DP}$ da distribuição e consumo entre 2 e 3 DP quando incompatível com atividade física e IMC.

Dos 3.959 adolescentes, 50,9\% eram do sexo feminino. Quanto à escolaridade, 54,7\% dos jovens tinham nove ou mais anos de estudo e em mais de um terço dos indivíduos a escolaridade do chefe da família ficou entre cinco e oito anos de estudo. Quanto às variáveis de estilo de vida, observou-se que $26,2 \%$ dos jovens possuíam o hábito de consumo de bebida alcoólica e 13,6\% o de fumar pelo menos uma vez na semana ( $\mathrm{Ta}-$ bela 1).

A média geral de pontos do IQD-R foi 62,4 com amplitude de 19,9 à 95,2 pontos e o desvio padrão de 12,0 (Tabela 2). As médias para as pontuações apresentaram-se mais baixas para os componentes "leite e derivados", "frutas totais", "vegetais totais", "vegetais verde escuro e alaranjados" e pontuações mais altas foram encontradas para os componentes "carne, ovos e leguminosas", "óleos", "sódio" e "Gord AA".

A pontuação do IQD-R foi maior nos adolescentes com cor de pele não branca, cujos chefes da família tinham menor escolaridade e que se encontravam no menor quintil do indice de bens (Tabela 3). Não se observou diferença nas médias dos escores entre os sexos, em relação à escolaridade do adolescente e ao estado nutricional. Em relação às variáveis de estilo de vida, tiveram menores escores de pontuação no IQD-R os adolescentes que fumavam e que consumiam bebida alcoólica. 
Tabela 1. Características dos participantes conforme variáveis sociodemográficas, de estado nutricional e estilo de vida. Pelotas, 2014. $(\mathrm{N}=4.106)$

\begin{tabular}{|c|c|c|}
\hline Variável & $\mathbf{N}$ & $\%$ \\
\hline \multicolumn{3}{|l|}{ Sexo $(N=4.106)$} \\
\hline Masculino & 2.015 & 49,1 \\
\hline Feminino & 2.091 & 50,9 \\
\hline \multicolumn{3}{|l|}{ Cor da pele $(\mathrm{N}=3.791)$} \\
\hline Branca & 2.526 & 66,6 \\
\hline Não branca & 1.265 & 33,4 \\
\hline \multicolumn{3}{|c|}{ Escolaridade (anos) $(\mathrm{N}=4.104)$} \\
\hline 0 a 4 & 196 & 4.8 \\
\hline 5 a 8 & 1.663 & 40,5 \\
\hline 9 ou mais & 2.245 & 54,7 \\
\hline \multicolumn{3}{|l|}{$\begin{array}{l}\text { Escolaridade do chefe da } \\
\text { família }(\mathrm{N}=3.751)\end{array}$} \\
\hline 0 a 4 & 969 & 25,8 \\
\hline 5 a 8 & 1301 & 34,7 \\
\hline 9 a 11 & 964 & 25,7 \\
\hline 12 ou mais & 517 & 13,8 \\
\hline \multicolumn{3}{|c|}{ Excesso de peso $(\mathrm{N}=3.961)$} \\
\hline Não & 2.881 & 72,7 \\
\hline Sim & 1.080 & 27,3 \\
\hline \multicolumn{3}{|c|}{ Consumo de álcool $^{*}(\mathrm{~N}=3.959)$} \\
\hline Não & 2.923 & 73,8 \\
\hline Sim & 1.036 & 26,2 \\
\hline \multicolumn{3}{|c|}{ Hábito de fumar ${ }^{* *}(\mathrm{~N}=3.958)$} \\
\hline Não & 3.420 & 86,4 \\
\hline Sim & 538 & 13,6 \\
\hline
\end{tabular}

Máximo de indivíduos com informação ignorada foi para variável escolaridade do chefe da família $(\mathrm{n}=355) .{ }^{*}$ consumo prejudicial de álcool baseado no questionário do The Alcohol Use Disorders Identification Test (AUDIT), com ponto de corte igual ou superior a 8 pontos. ${ }^{* *}$ Hábito atual de fumar pelo menos uma vez por semana.
A Tabela 4 apresenta a frequência de jovens que atingiram a recomendação do Guia Alimentar para População Brasileira para os componentes do IQD-R.

\section{Discussão}

A média do IQD-R neste estudo foi de 62,4 pontos. Observando outros estudos com adolescentes que avaliaram a qualidade da dieta utilizando índices, constata-se que a média encontrada neste foi superior, tanto comparado aos feitos no Brasil $^{19-21}$ como no exterior ${ }^{22,23}$.

No Brasil, Assumpção et al. ${ }^{21}$ avaliaram o IQD adaptado de Godoy et al. ${ }^{20}$ em amostra de 409 adolescentes, encontrando uma pontuação média de 59,7 pontos, com pontuações baixas para os componentes verduras e legumes, frutas e leite e derivados. Estudo conduzido por Godoy et al. ${ }^{20}$ no Distrito do Butantã, em São Paulo, utilizou o mesmo índice e obteve pontuação média de 58,4 pontos, sem diferença estatisticamente significativa entre os sexos e escolaridade do chefe da família. Já Andrade et al. ${ }^{19}$, ao estudar adolescentes que viviam no estado de São Paulo, encontrou uma pontuação média de 59,7, com médias de escores mais baixos para os componentes frutas, laticínios e legumes.

Acar Tek et al..$^{22}$, na Turquia, em estudo transversal com uma amostra de 1.104 adolescentes, avaliaram a qualidade da dieta através do HEI, obtendo o valor médio de 51,5 pontos. Mariscal -Arcas et al. ${ }^{23}$, na Espanha, realizaram um estudo transversal com amostra de 288 indivíduos com idades entre 6 e 18 anos, obtendo média de 56,3

Tabela 2. Média do IQD-R e dos componentes. Pelotas, 2014. ( $\mathrm{N}=3.959)$

\begin{tabular}{|c|c|c|c|c|}
\hline Variáveis & Pontuação Máxima & Média (Pontos) & $\mathrm{DP}^{*}$ & Mediana \\
\hline IQD-R total & 100 & 62,4 & 12,0 & 62,7 \\
\hline Frutas totais & 10 & 3,7 & 3,5 & 2,3 \\
\hline Vegetais totais & 5 & 3,4 & 1,8 & 4,4 \\
\hline $\begin{array}{l}\text { Vegetais verdes escuros e alaranjados e leguminosas } \\
\text { escuros e alaranjados }\end{array}$ & 5 & 3,0 & 2,0 & 3,6 \\
\hline Cereais totais & 10 & 5,2 & 3,0 & 4,0 \\
\hline Leite e derivados & 10 & 3,6 & 3,0 & 2,8 \\
\hline Carnes, ovos e leguminosas & 10 & 7,7 & 2,5 & 8,4 \\
\hline Óleos & 10 & 9,9 & 0,1 & 10 \\
\hline Gordura saturada & 10 & 6,9 & 2,7 & 7,8 \\
\hline Sódio & 10 & 9,3 & 1,1 & 9,9 \\
\hline Gord_AA ${ }^{* *}$ & 20 & 9,6 & 8,1 & 10,6 \\
\hline
\end{tabular}

DP- Desvio Padrão. ${ }^{* *}$ Gord_AA: calorias provenientes de gordura sólida (saturada e trans, álcool e açúcar de adição) . 


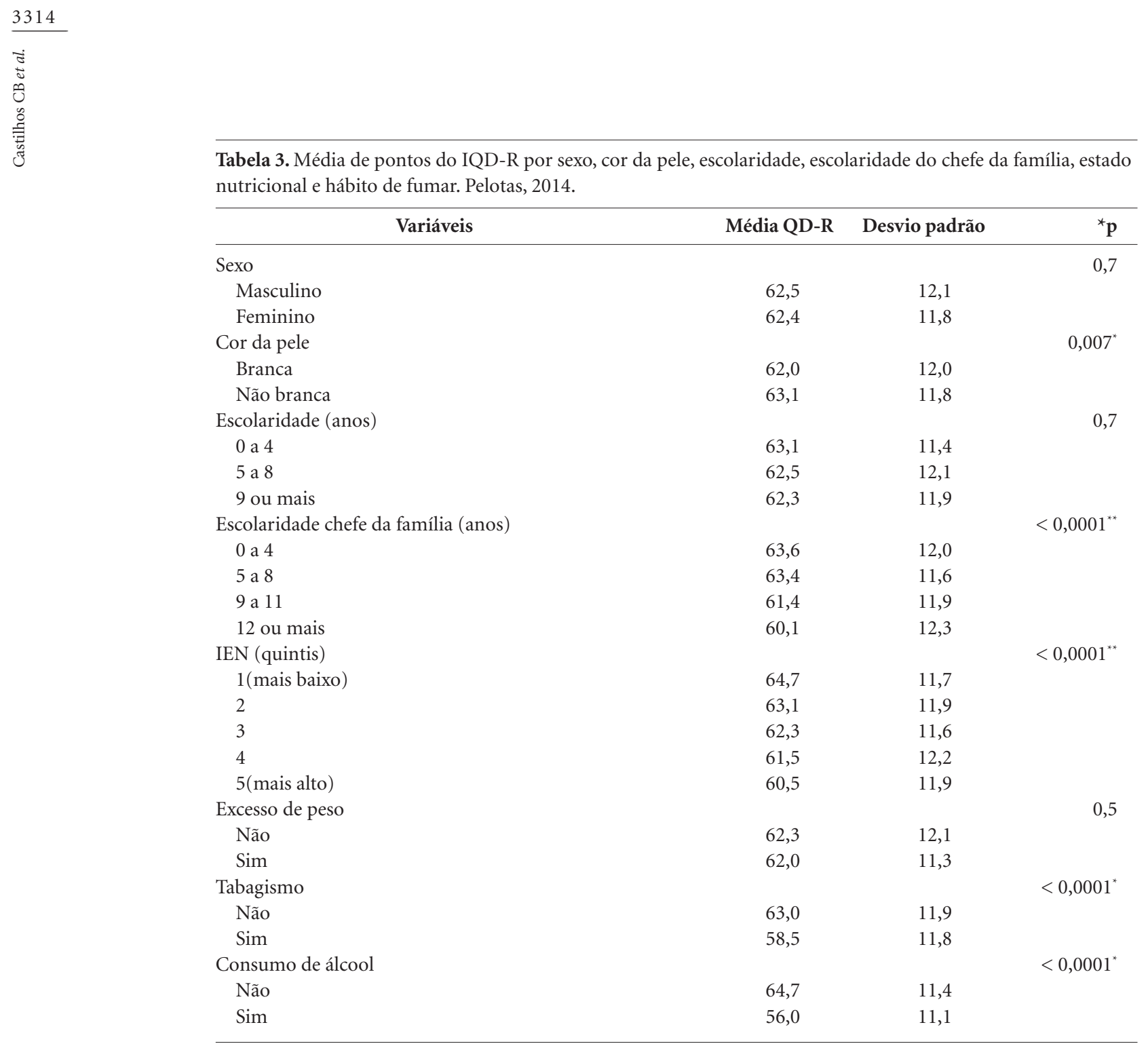

${ }^{*}$ Teste de Wald. ${ }^{* *}$ Teste $\mathrm{t}$ de Student.

Tabela 4. Frequência de jovens que atingiram a recomendação do Guia Alimentar para População Brasileira para os componentes do IQD-R. Pelotas. 2014. $(\mathrm{N}=3.959)$

\begin{tabular}{lrrrrr}
\hline \multirow{2}{*}{ Componentes } & \multicolumn{3}{c}{ Atingiu recomendação } \\
\cline { 2 - 3 } \cline { 6 - 7 } & \multicolumn{2}{c}{ Sim } & & \multicolumn{2}{c}{ Não } \\
\cline { 2 - 3 } \cline { 6 - 7 } & \multicolumn{1}{c}{$\mathbf{n}$} & \% & & n & $\%$ \\
\hline Frutas totais & 856 & 21,6 & & 3.103 & 78,4 \\
Vegetais totais & 438 & 11,1 & & 3.521 & 88,9 \\
Cereais Totais & 1.060 & 26,8 & & 2.899 & 73,2 \\
Leite e derivados & 238 & 6,0 & & 3.721 & 94,0 \\
Carnes e ovos & 1.322 & 33,4 & & 2.637 & 66,6 \\
Oleos & 3.954 & 99,9 & & 5 & 0,1 \\
\hline
\end{tabular}

pontos no escore do Diet Quality Index International (DQI-I).

Em relação aos componentes do IQD-R, os adolescentes obtiveram escores mais baixos para "leite e derivados", "frutas totais", "vegetais verde escuro e alaranjados" e "vegetais totais". Tais achados são compatíveis com os encontrados por Wendpap ${ }^{24}$ em estudo realizado com adolescentes de Cuiabá utilizando o IQD-R e também com aquele conduzido por Neutzling et al. ${ }^{25} \mathrm{em}$ Pelotas, em 2004, com escolares de 13 e 14 anos de idade. Neutzling et al. ${ }^{25}$ observaram que somente $5,3 \%$ dos adolescentes consumiam vegetais ou frutas na frequência desejada (cinco ou mais vezes por dia).

Ao analisar o comportamento dos adolescentes quanto ao consumo de leite e derivados, observou-se que somente $6 \%$ dos jovens conso- 
mem 1,5 porção ao dia, conforme recomendação do guia alimentar. Este achado mostrou concordância com os estudos conduzidos por Ramos ${ }^{26}$, Pinheiro e Atalah ${ }^{27}$ e Andrade et al..$^{19}$. Já Neutzling et al. ${ }^{25}$, na cidade de Pelotas, encontraram que metade dos jovens consumia leite diariamente.

Observando os componentes "cereais totais" (média de 5,2 pontos) e "carnes, ovos e leguminosas" (média de 7,7 pontos) percebe-se que respectivamente $26,8 \%$ e $66,6 \%$ dos jovens alcançam as recomendações do guia alimentar. Já em relação ao componente "óleos", quase a totalidade dos adolescentes encontra-se na faixa de consumo considerada adequada. Porém, uma das limitações deste estudo é a impossibilidade de estimar a quantidade do óleo utilizado para fins culinários. Isto pode ter afetado a pontuação deste componente e tambem a total, subestimando-a.

Ainda em relação ao componente "carnes, ovos e leguminosas", os escores mais altos para a média de pontuação foram também encontrados por Ramos ${ }^{26}$ em estudo com adolescentes na cidade de Niterói/RJ utilizando o IQD-R. Acredita-se que a alta pontuação pode ser atribuida à inclusão do grupo das leguminosas a este componente, visto que o consumo de feijão é bastante frequente na alimentação da população brasileira $^{28}$. Sugere-se que ocorra uma revisão na construção do IQD-R quanto ao procedimento de inclusão do grupo das leguminosas no grupo das carnes e ovos.

Quanto ao componente gordura saturada (média de 6,9 pontos) a amostra apresentou consumo entre $10 \%$ e $15 \%$ do Valor Energético Total (VET). A maior pontuação (10 pontos) para este componente representava consumo adequado, ou seja, $\leq 7 \%$ do VET. Este achado é contrário ao encontrado por Diethelm et al. ${ }^{29}$ em 2011, ao estudar uma amostra de escolares residentes em dez cidades europeias, participantes do Healthy Lifestyle in Europe by Nutrition in Adolescence Study (HELENA), com consumo 2,5 vezes maior do que a recomendação.

Ao observar o comportamento dos jovens quanto ao componente Gord_AA (calorias provenientes de gordura sólida [saturada e trans], álcool e açúcar de adição), pode-se observar uma média de 9,6 pontos. Para este componente a pontuação máxima poderia atingir 20 pontos, o que corresponderia a $10 \%$ do VET. No entanto, este escore pode estar subestimado devido a não inclusão das gorduras trans no cálculo do componente, uma vez que a maioria dos alimentos não tinham tal informação nas tabelas de com- posição nutricional utilizadas. Em estudo semelhante, utilizando o mesmo índice, com amostra de adolescentes, na cidade de Cuiabá, Wendpap ${ }^{24}$ encontrou média de 18,6 pontos, muito superior a encontrada no presente estudo, porém sem avaliar o consumo de álcool.

A média do escore para o item sódio foi de 9,3 pontos, revelando um consumo acima da recomendação que é de $\leq 0,75 \mathrm{~g} / 1000 \mathrm{Kcal}$. Este achado é semelhante ao observado em outros estudos brasileiros sobre o consumo alimentar de adolescentes $^{19,24,26}$. Possivelmente o consumo elevado de sódio esteja ligado ao de alimentos processados, visto que, $25,3 \%$ dos jovens consumiam embutidos (mortadela, presunto, salame) pelo menos uma vez por dia (dados não apresentados).

Analisando as variáveis socioeconômicas, observa-se que a qualidade da dieta diminui à medida que aumenta a escolaridade do chefe da família e que a média de pontos do IQD-R foi significativamente menor para os adolescentes que se encontram no maior quintil do índice de bens. Tais achados podem estar vinculados à diminuição da influência dos pais e do ambiente familiar nas escolhas alimentares dos jovens cujos pais têm maior escolaridade e maior renda. Ainda, é possível que os adolescentes, especialmente aqueles cujas famílias estejam no mais alto quintil de IEN, substituam alimentos saudáveis como frutas e legumes por lanches e doces, fenômeno prevalente nessa faixa etária. Também pode estar ocorrendo um aumento do consumo de alimentos fora de casa e de fast food em jovens das classes econômicas mais abastadas ${ }^{30}$.

Segundo Wang et al. ${ }^{31}$, os adolescentes pertencentes a famílias cujo chefe é mais escolarizado são mais predispostos a ter uma dieta com baixo nível de ingestão de frutas e vegetais e rica em gorduras.

Os resultados deste estudo apontam relação positiva entre a qualidade da dieta dos adolescentes e características do estilo de vida. O IQD-R foi maior nos adolescentes que não ingerem bebida alcoólica e que não fumavam. Estudo realizado por Guenther et al..$^{32}$ nos Estados Unidos em 2008, com individuos com 20 anos ou mais, encontrou pontuação menor de HEI e seus componentes para os fumantes. Resultado semelhante foi encontrado por McNaughton et al. ${ }^{33} \mathrm{em}$ estudo transversal com amostra de adultos com idade $\geq 19$ anos na Austrália em 2008. Em relação à ingestão de bebida alcoólica, não fomos capazes de explicar este achado, pois não encontramos outros estudos que avaliassem seu consumo relacionado à qualidade da dieta em adolescentes. 
As principais limitações do estudo se referem ao uso do QFA, o qual apesar de estimar o consumo alimentar usual ao longo de um período, pode levar à superestimação do consumo ${ }^{34}$. Somado a isso, o QFA utilizado neste estudo não contemplou questões sobre a ingestão de cereais integrais e óleo de uso culinário. Como limitação do índice pode-se citar o fato de não haver distinção do grupo "leguminosas", já que o feijão é um alimento típico da dieta do brasileiro. O IQD-R aparece como um instrumento para avaliar o padrão de consumo alimentar, porém a ausência de limite superior para consumo dos componentes da dieta não permite analisar o consumo energético total.

Um ponto positivo do IQD-R é que o mesmo utiliza como recomendação o guia alimentar para a população brasileira de $2006^{11}$, o qual per- mite avaliar a aderência da dieta às recomendações nutricionais propostas para os vários estágios de vida.

Concluindo, este estudo mostrou que a qualidade da dieta dos adolescentes, pertencentes à coorte de 1993, na cidade de Pelotas, medida através do IQD-R, necessita de atenção. Menores escores foram encontrados para frutas, leite $\mathrm{e}$ produtos lácteos e verduras e os mesmos estiveram associados a variáveis socioeconômicas e de estilo de vida. O estímulo à adoção de hábitos alimentares saudáveis, através do desenvolvimento e aperfeiçoamento de programas de intervenção nutricional, especialmente em escolas e universidades, poderá promover mudanças no comportamento alimentar prevenindo futuras doenças crônicas não transmissíveis.

\section{Colaboradores}

CB Castilhos, BC Schneider, LC Muniz e MCF Assunção leram e aprovaram o conteúdo do manuscrito e contribuíram em todas as etapas desse trabalho. 


\section{Referências}

1. World Health Organization (WHO). Nutrition in adolescence: issues and challenges for the health sector. Geneva: WHO; 2006.

2. Levy RB, Castro IRR, Cardoso LO, Tavares LF, Sardinha LMV, Gomes FS, Costa AWN. Consumo e comportamento alimentar entre adolescentes brasileiros: Pesquisa Nacional de Saúde do Escolar (PeNSE), 2009. Cien Saude Colet 2010; 15(Supl. 2):3085-3097.

3. Instituto Brasileiro de Geografia e Estatística (IBGE). Censo demográfico: resultados gerais da amostra. Rio de Janeiro: IBGE; 2011.

4. Nunes MMA, Figueiroa JN, Alves JGB. Excesso de peso, atividade física e hábitos alimentares entre adolescentes de diferentes classes econômicas em Campina Grande (PB). Rev Assoc Med Bras 2007; 53(2):130-134.

5. Toral N, Conti MA, Slater B. A alimentação saudável na ótica dos adolescentes: percepções e barreiras à sua implementação e características esperadas em materiais educativos. Cad Saude Publica 2009; 25(11):2386-2394.

6. Santos JS, Costa MCO, Nascimento Sobrinho CL, Silva MCM, Souza KEP, Melo BO. Perfil antropométrico e consumo alimentar de adolescentes de Teixeira de Freitas - Bahia. Rev Nutr 2005; 18(5):623-632.

7. Fisberg RM, Slater B, Barros RR, Lima FD, Cesar CLG, Carandina L, Barros MBA, Goldbaum M. Índice de Qualidade da Dieta: avaliação da adaptação e aplicabilidade. Rev Nutr 2004; 17(3):301-318.

8. Cervato AM, Vieira VL. Índices dietéticos na avaliação da qualidade global da dieta. Rev Nutr 2003; 16(3):347355.

9. Previdelli AN, Andrade SC, Pires MM, Ferreira SR, Fisberg RM, Marchioni DM. A revised version of the Healthy Eating Index for the Brazilian population. Rev Saude Publica 2011; 45(4):794-798.

10. Guenther PM, Reedy J, Krebs-Smith SM. Development of the Healthy Eating Index-2005. J Am Diet Assoc 2008; 108(11):1896-1901.

11. Brasil. Ministério da Saúde (MS). Coordenação-Geral da Política de Alimentação e Nutrição. Guia alimentar para a população brasileira: promovendo a alimentação saudável. Brasília: MS; 2006. (Série A. Normas e Manuais Técnicos)

12. Vieira VCR, Priore SE, Ribeiro SMR, Franceschini SCC, Almeida LP. Perfil socioeconômico, nutricional e de saúde de adolescentes recém-ingressos em uma universidade pública brasileira. Rev Nutr 2002; 15(3):273282.

13. Victora CG, Araújo CL, Menezes AM, Hallal PC, Vieira Mde F, Neutzling MB, Gonçalves H, Valle NC, Lima RC, Anselmi L, Behague D, Gigante DP, Barros FC. Metodological aspects of the 1993 Pelotas (Brazil) Birth Cohort Study. Rev Saude Publica 2006; 40(1):39-46.

14. Victora CG, Hallal PC, Araújo CL, Menezes AM, Wells JC, Barros FC. Cohort Profile: The 1993 Pelotas (Brazil) Birth Cohort Study. Int J Epidemiol 2008; 37(4):704-709.

15. Gonçalves H, Assunção MC, Wehrmeister FC, Oliveira IO, Barros FC, Victora CG, Hallal PC, Menezes AM. Cohort Profile update: The 1993 Pelotas (Brazil) Birth Cohort follow-up visits in adolescence. Int J Epidemiol 2014; 43(4):1082-1088.
16. Fabor TF, Higgins-Biddle JC, Saunders JB, Monteiro MG. The Alcohol Use Disorders Identification Test (AUDIT) - Guidelines for Use in Primary Care. Geneva: WHO; 2001.

17. Barros AJD, Victora CG. Indicador econômico para o Brasil baseado no censo demográfico de 2000. Rev Saude Publica 2005; 39(4):523-529.

18. Núcleo de Estudos e Pesquisa em Alimentação (NEPA). Tabela Brasileira de Composição de Alimentos (TACO). $4^{\text {a }}$ ed. Campinas: Unicamp; 2011.

19. Andrade SC, Azevedo Barros MB, Carandina L, Goldbaum M, Cesar CL, Fisberg RM. Dietary Quality Index and Associated Factors among Adolescents of the State of Sao Paulo, Brazil. J Pedr 2010; 156(3):456-460.

20. Godoy FC, Andrade SC, Morimoto JM, Carandina L, Goldbaum M, Barros MBA, Cesar CLG, Fisberg RM. Índice de qualidade da dieta de adolescentes residentes no distrito do Butantã, município de São Paulo, Brasil. Rev Nutr 2006; 19(6):663-671

21. Assumpção Dd, Barros MBA, Fisberg RM, Carandina L, Goldbaum M, Cesar CLG. Qualidade da dieta de adolescentes: estudo de base populacional em Campinas, SP. Rev bras epidemiol 2012; 15(3):605-616.

22. Acar Tek N, Yildiran H, Akbulut G, Bilici S, Koksal E, Gezmen Karadag M, Sanlier N. Evaluation of dietary quality of adolescents using Healthy Eating Index. Nutr Res Pract 2011; 5(4):322-328.

23. Mariscal-Arcas M, Romaguera D, Rivas A, Feriche B, Pons A, Tur JA, Olea-Serrano F. Diet quality of young people in southern Spain evaluated by a Mediterranean adaptation of the Diet Quality Index-International (DQI-I). Br J Nutr 2007; 98(6):1267-1273.

24. Wendpap LL. Qualidade da dieta de adolescentes e fatores associados. Cad Saude Publica 2014; 30(1):97106

25. Neutzling MB, Assunção MCF, Malcon MC, Hallal PC, Menezes AMB. Hábitos alimentares de escolares adolescentes de Pelotas, Brasil. Rev Nutr 2010; 23(3):379388.

26. Ramos JM. Índice de qualidade da dieta e indicadores do estado nutricional de adolescentes assistidos no Programa Médico de Família em Niterói-RJ [dissertação]. Niterói: Universidade Federal Fluminense; 2010.

27. Pinheiro FAC, Atalah E. Propuesta de una metodología de análisis de la calidad global de la alimentación. Rev Med Chile 2005; 133(2):175-182.

28. Instituto Brasileiro de Geografia e Estatística (IBGE). Pesquisa de Orçamentos Familiares 2008-2009: análise do consumo alimentar pessoal no Brasil. Rio de Janeiro: IBGE; 2011.

29. Diethelm K, Jankovic N, Moreno LA, Huybrechts I, De Henauw S, De Vriendt T, González-Gross M, Leclercq C, Gottrand F, Gilbert CC, Dallongeville J, Cuenca-Garcia M, Manios Y, Kafatos A, Plada M, Kersting M; HELENA Study Group. Food intake of European adolescents in the light of different food-based dietary guidelines: results of the HELENA (Healthy Lifestyle in Europe by Nutrition in Adolescence) Study. Public Health Nutr 2012; 15(3):386-398. 
30. Nielsen SJ, Siega-Riz AM, Popkin BM. Trends in Food Locations and Sources among Adolescents and Young Adults. Prev Med 2002; 35(2):107-113.

31. Wang Y, Bentley ME, Zhai F, Popkin BM. Tracking of Dietary Intake Patterns of Chinese from Childhood to Adolescence over a Six-Year Follow-Up Period. J Nutr 2002; 132(3):430-438.

32. Guenther PM, Reedy J, Krebs-Smith SM, Reeve BB. Evaluation of the Healthy Eating Index-2005. J Am Diet Assoc 2008; 108(11):1854-1864.

33. McNaughton SA, Ball K, Crawford D, Mishra GD. An index of diet and eating patterns is a valid measure of diet quality in an Australian population. J Nutr 2008; 138(1):86-93.

34. Willett WC. Future directions in the development of food-frequency questionnaires. Am J Clin Nutr 1994; 59(1):171S-174S.

Artigo apresentado em 05/11/2014

Aprovado em 28/05/2015

Versão final apresentada em 30/05/2015 